\title{
A Self-Tuning procedure for resource management in InterCloud Computing
}

\author{
Suleiman Onimisi Aliyu, Feng Chen \\ Software Technology Research Laboratory (STRL), \\ De Montfort University \\ Leicester, UK \\ soaliyu@hotmail.com, fengchen@dmu.ac.uk
}

\author{
Han Li \\ Beijing Key Laboratory on Integration and Analysis of \\ Large-scale Stream Data, College of Computer Science, \\ North China University of Technology, Beijing, China \\ hanli@ncut.edu.cn
}

\begin{abstract}
InterCloud Computing is a new cloud paradigm designed to guarantee service quality or performance and availability of on-demand resources. InterCloud enables Cloud interoperability by promoting the interworking of Cloud systems from different cloud providers using standard interfacing. Resource management in InterCloud, considered as an important functional requirement, has not attracted commensurate research attention. The focus of this paper is to propose a Software Cybernetic approach, in the form of an adaptive control framework, for efficient management of shared resources in peer-to-peer InterCloud computing. This research effort adopts cooperative game theory to model resource management in InterCloud. The space of cooperative arrangements (resource sharing) between the participant cloud systems is presented by using Integer Partitioning to characterise the worst case communication complexity in peer to peer InterCloud. Essentially, this paper presents an Integer partition based anytime algorithm as an optimal cost solution to the bi-objective optimisation problem in resource management; anchored principally on practical trade-off between the desired performance (quality of service) and communication complexity of collaborating resource clouds.
\end{abstract}

Keywords-Software Cybernetics; Multi-objective optimisation; Adaptive control; Cooperative game theory; Resource management; InterCloud Computing.

\section{INTRODUCTION \& BACKGROUND}

$\mathrm{C}$ loud computing offers on-demand access to a shared pool of resources e.g. storage, processors, Virtual machines and network bandwidth. In today's Cloud paradigm, Consumers request and utilise resources via services from monolithic Cloud systems (e.g. Amazon web services, Rackspace, Microsoft and Google). These Cloud service providers (CSPs) achieve economies of scale for shared resources by delivering their resources as a utility (similar to electric power, gas or water) in a pay-as-you-go manner. Although Cloud computing promises the illusion of infinite resources (Toosi et al., 2014), essentially, the ability of a single cloud system to handle requests for reserved resources is bounded by limits (Aoyama and Sakai, 2011). For Instance, the Google Prediction API (Google cloud platform, 2015) stipulates 2.5 GB is the maximum size of a text file data source for Google's Machine learning-as-a-service.
Furthermore, Conventional Cloud computing systems may be insufficient to support Internet of Things (IoT) or big data applications which produce and process vast amounts of data. Unexpected spikes in loads and service failures may overbear a cloud system leading to interrupted services or unreliability (Aoyama and Sakai, 2011; Toosi et al., 2014). Moreover, it is highly improbable for proprietary cloud infrastructure providers to establish data centers at all possible locations (e.g. North America, Europe, South-east Asia) to meet the lowlatency requirements of consumer applications. Consequently, it has become imperative to adapt distributed resources (across clouds) in response to load variations (Buyya et al., 2010). Cloud interoperability is largely seen as a panacea to most of the foreseeable problems of the current cloud model. However, it is worthwhile to note that interoperable clouds come with inherent challenges.

Vendor lock-in, a situation in which consumers can't easily migrate between vendors, is seen as one of the drawbacks of the extant cloud computing models (Armbrust et al., 2009). Vendor lock-in in Cloud computing arises as direct consequence of cloud non-compatibility or lack of interoperability. Cloud interoperability demands Cloud Providers cooperate by adopting and implementing standard protocols, components and interfaces for their proprietary resources (Toosi et al., 2014). In practice, Cloud interoperability can be enabled either though service brokering or by using standard communication interfaces (Chen and Doumeingts, 2003; Toosi et al., 2014). Leading providercentric Cloud interoperability scenarios include Hybrid Clouds, Cloud Federation and InterCloud or 'cloud of clouds' (Banafa, 2014). Hybrid-Cloud by definition is a composition of two or more cloud infrastructures (e.g. a private and public cloud), which often use Cloud bursting- the use of public cloud resources when the private clouds are inadequate. Although some experts opt to use InterCloud and Cloud Federation synonymously in their work, others prefer to differentiate between the two terms. Specifically, Cloud Federation uses a provider (vendor) specification of the standard interfaces whereas Inter-Clouds is based on open technology and future standards. The Global Inter-cloud technology forum (GICTF) defines InterCloud computing in their whitepaper (GICTF, 2010) as a cloud model that 
guarantees performance and availability of each service, by allowing on-demand reassignment of resources and transfer of workload through interworking of cloud systems and use of standard interfaces. InterCloud can be classified generally into two broad groups: Volunteer federation and Multi-clouds (Grozev et al., 2012). Volunteer federation is a situation whereby group of cloud providers willingly or voluntarily agree to cooperate and share or exchange their resources (e.g. governmental clouds) while Multi-cloud occurs when multiple clouds are used in aggregation by an application or its broker. Based on architecture, Volunteer federations can further be classified into peer to peer or centralised. In the Centralised paradigm, a central entity is responsible for facilitating resource allocation whereas for peer to peer architecture, negotiation and cooperation is carried out directly between participatory clouds. Although resource management in InterCloud is still a relatively novel concept, the GICTF in their whitepaper (GICTF, 2010), regard resource management as an important functional requirement for InterCloud. According to Aoyama and Sakai (2011), an implementation of resource management for InterCloud must include a unified way (e.g. standard interfaces) of managing various resource configurations for each service such as servers, storage units and networks. They suggest the aim of this function is to manage resource configurations necessary to prevent services from degradation or disaster. Moreover, to ensure the reliability of web-based or Cloud services requires the enforcement of a testing process (Bai et al, 2007).

Software Cybernetics, described broadly in scope, is an area of research that explores the interplay between Software and control. Research in the field of Software Cybernetics is justified by new topics or theories that arise as a result of new control requirements, for example, quality of service (QoS) for Network Software (Cai et al, 2003). In this paper, our research effort is aimed at proposing a Cybernetic framework, comprising autonomic components, for efficient resource management in peer to peer volunteer InterCloud. Because of interdependencies that may exist between different collaborating cloud systems (resource coalitions), resource management for peer to peer InterCloud is modeled as partition function game in positive and negative settings, henceforth denoted $P F G+$ and $P F G-$ settings. In addition to performance of peer to peer federations in InterCloud, our study also considers communication complexity in order to minimise redundant message exchanges via standard interfacing. Minimising communication helps to save the operation cost and as a consequence increases throughput (efficiency) of the InterCloud computing environment. The main thrust of this paper then is to develop an anytime procedure to solve the coalition structure generation (CSG) problem of $\mathrm{p} 2 \mathrm{p}$ InterCloud resource management in $P F G+$ and $P F G$ - settings. Anytime algorithms are 'so called' because they can be terminated at any point during execution and return a 'good solution'. This class of algorithms record monotonic increments in the quality of the solution before termination. Another desirable property of our proposed anytime algorithm is search cost criterion. This is quite pragmatic for real world systems, as it would be most beneficial to improve the solution quality by searching paths of minimum cumulative cost. The rest of this paper is organised as follows. Sections II \& III detail the building blocks of our model for resource management in InterCloud Computing. Section IV formulates the multi-criteria optimisation problem and outlines our proposed algorithmic solution. Conclusion and our future work is provided in section $\mathrm{V}$.

\section{FRAMEWORK FOR RESOURCE MANAGEMENT IN PEER TO PEER INTER-CLOUD}

Forming coalitions is important for solving many distributed applications (e.g. ubiquitous computing) where adaptation to changing resources and environments is necessary (Li, 2007). Horling and Lesser (2004) specified the important characteristics of coalitions that distinguish them from other organisations. According to their specification, "Coalitions in general are goal-oriented and short-lived; they are formed with a purpose in mind and dissolve when that purpose no longer exists". Coalition formation process can generally be considered to include three main activities: (i) compute the value of every possible coalition that can be formed. (ii) Compute the set of disjoint coalitions that have maximum total utility. (iii) Determine the reward each member agent gets in a coalition (Rahwan, 2007). Note that, sharing the reward is not essential in cooperative games as players are mainly concerned with maximising social welfare.

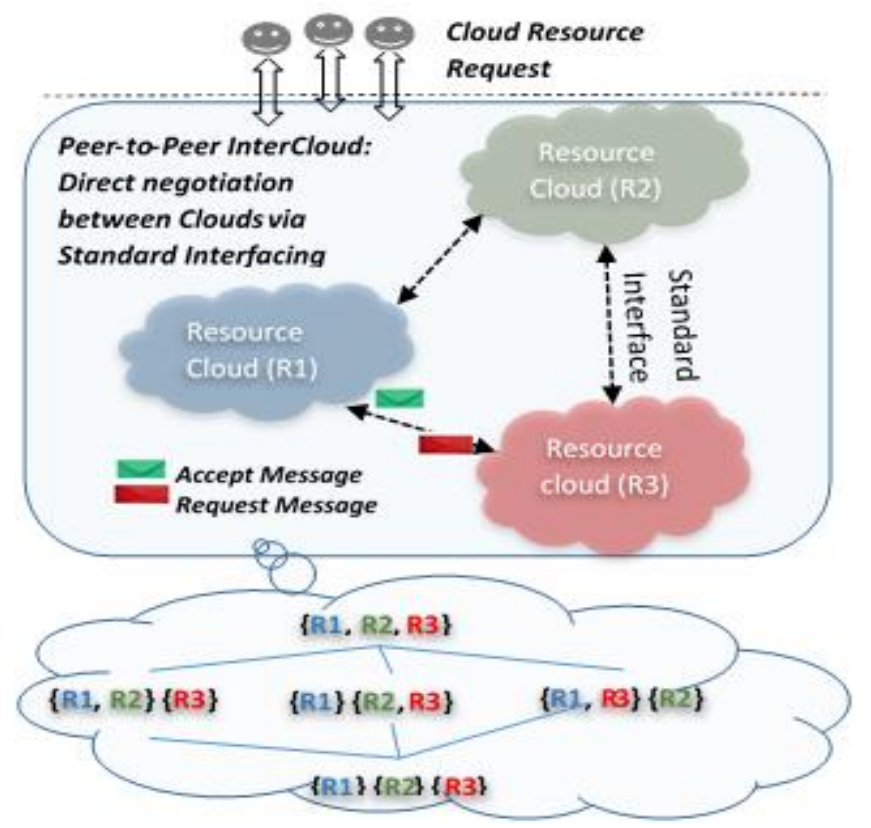

Figure. 1. Peer to Peer InterCloud architecture - direct negotiation between 3 volunteer cloud systems.

Formally, our effort models management of resources in peer-to-peer (p2p) InterCloud, shown in Figure 1, as a cooperative (coalition) game. Let the set $A$ be the set of distinct (singleton) resource pools representing resources (e.g. VMs, storage, networks) from $q$ participating cloud providers 
$\left\{p_{1}, p_{2}, p_{3}, \ldots, p_{q}\right\}$ in the Inter-cloud computing environment, i.e. $A=\left\{R_{1}, R_{2}, R_{3}, \ldots, R_{q}\right\}$. Then by definition, a resource coalition $C$ is defined as a subset of $A(C \subset A)$. The set of all possible resource coalitions is given by $2^{q}$. By convention, we also talk of the empty set $(\varnothing)$ as a coalition, the empty coalition. The set $A$ is also a resource coalition, called the grand resource coalition comprising resource pools from all $q$ cloud providers. To increase Cloud resource types or capacity, we propose cloud providers in peer to peer InterCloud collaborate via sharing resources (horizontal federation) and form resource coalitions. As such, the subset $C_{\mathrm{i}}$ of $A$, called a feasible resource coalition is given as:

$$
C_{\mathrm{i}}=\left\{R_{i} \mid i \subseteq\{1,2 \ldots q\}\right\}
$$

The coalition structure generation (CSG) problem, an important activity of coalition formation, involves partitioning the set of players into exhaustive and disjoint coalitions (see (2) and (3)) so as to maximise social welfare (Sandholm et al, 1999; Rahwan, 2007). More so, in a number of real world environments, there are significant externalities from coalition formation in which the performance of one coalition may be affected by the formation of another distinct coalition (Michalak, 2008). For instance, in a context such as purchasing Cloud resources, ad hoc coalition formation will need to allow for coalition externalities in as broad a sense as possible (Rahwan, 2009). Hence, characteristic function games (CFGs) would be considered inadequate to represent such environments. In contrast to CFGs, Partition form games (PFGs) enable a representation that accounts for externalities. As described by Hafalir (2007), a partition form game (PFG) has positive (or negative) externalities if a merger between two coalitions may improve (or worsen) the performance of other coalitions.

\section{A. Partition Form Games (PFGs) for resource management in peer to peer InterCloud}

A resource coalition structure or resource structure $(R S)$ in peer to peer InterCloud is defined as a partition (comprising resource coalitions, see (1)) of $\mathrm{A}$, that is, $R S=\left\{C_{1}, C_{2}, \ldots\right.$, $\left.C_{k}\right\}$, where $1 \leq k \leq q$ ), hence,

$$
\begin{aligned}
& C_{\mathrm{n}} \neq \emptyset, \text { for any } \mathrm{n} \in 1,2 \ldots k \text { and } \bigcup_{n=1}^{k} C_{n}=A \\
& C_{l} \cap C_{h}=\emptyset, \text { for any } l, h \in 1,2 \ldots k \text { and } l \neq h
\end{aligned}
$$

Mergers of resource coalitions in InterCloud may have a positive impact on the value of other resource coalitions in Inter-cloud especially when resource pools are degraded or experiencing major failures. On the other hand, the performance of resource coalitions could be hampered as a result of the increased cooperation (communication complexity) of other coalitions, particularly if resources are readily available and adequate. Consequently, PFG's for resource management in peer to peer InterCloud can exhibit either positive or negative externalities.

In games with externalities for resource management in InterCloud, the payoff (value) of a resource coalition may change as the resource coalition structure which contains it changes. This means that it is not generally possible to predetermine the reward of a resource coalition in a certain coalition structure without actually computing it in that specific structure. As a result, finding an optimal resource configuration (or resource structure) can only be guaranteed by searching through the entire search space of resource structures. This can easily be classified an NP-hard problem if the search space were too large to be fully searched (Guazzone et al, 2014; Rahwan, 2007). Consequently, our solution adopts the class of anytime algorithms to generate solutions that although not optimal, are guaranteed to be within a bound from the optimal, and is established by searching partial subsets of the space. Additionally, an optimal solution can be guaranteed with further search (if time permits). A Partition form game for resource management in Inter-Cloud consists of the set of resource pools $A$ and a multi-valued partition function which takes as input, every feasible resource coalition structure $R S$, and for every resource coalition $(C)$ in that structure, outputs a set-valued payoff that reflects the performance of the resource coalition in that structure. The set-valued payoff for a resource coalition represents the success rate of matching Cloud consumer's request to resources, i.e. comprising the number of successful matches and number of request attempts, it achieves in a specific resource coalition structure. Furthermore, computing the performance of resource coalitions is carried out (in an efficient manner) using the distributed coalition value calculation (DCVC) algorithm as presented in Rahwan (2007).

\section{B. Resource Structure Graph}

In its simplest form, the solution to the resource structure generation problem can be described as a search in a coalition structure graph (Sandhlom et al, 1999), a graph in which every node represents a coalition structure. The resource structure graph for peer to peer Inter-cloud is based on a novel representation of the search space called Integer partition (IP) graph (Rahwan, 2012). Using this representation, every node (called provider or resource node) in the resource graph represents a subspace that comprises feasible resource coalition structures, nodes are categorised into $k$ levels (partition spaces) denoted as $I_{1}, I_{2}, \ldots, I_{k}$. Partition space $I_{n}$ contains the resource structures comprising $n$ resource coalitions. For instance, if the number of cloud providers or 'singleton' resource coalitions in InterCloud is $k=6$, then the search space is the set $G$ of all integer partitions (partition spaces) of $k$ is given as,

$$
\begin{aligned}
& G=\{\{6\},\{3,3\},\{2,4\},\{1,5\}\{2,2,2\},\{1,2,3\},\{1,1,4\}, \\
& \{1,1,2,2\},\{1,1,1,3\},\{1,1,1,1,2\},\{1,1,1,1,1,1\}\}
\end{aligned}
$$

Definition: A resource structure graph (or simply resource graph) is an Integer partition graph in which the provider nodes (subspaces) on each level are ordered in ascending or descending order of the maximum resource coalition size it contains (see Fig. 2). Arcs in the resource structure graph represents mergers (more communication between providers 
using standard interfaces) of resource coalitions when followed upwards and splits of resource coalitions (less communication between providers using standard interfaces) when followed downwards.

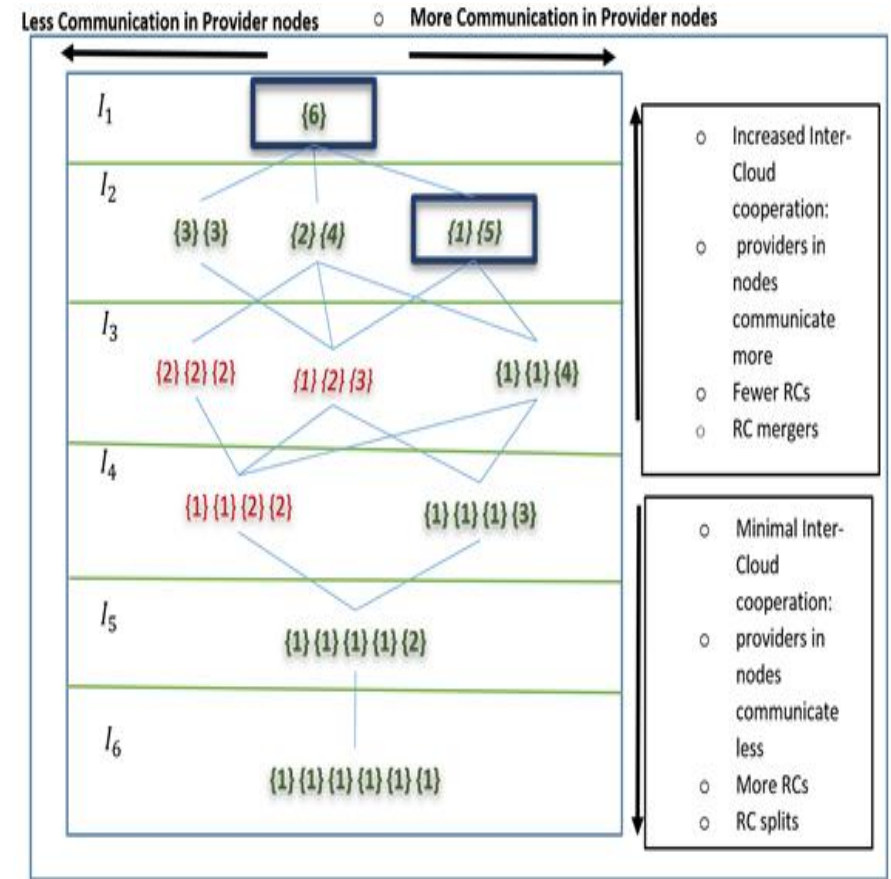

Figure. 2. Resource structure graph (or simply resource graph) for 6 resource Clouds (RCs).

\section{COMMUNiCATION COMPLEXITY IN PEER TO PEER INTERCLOUD COMPUTING}

We refer to each Cloud provider's pool of resources in peer to peer InterCloud computing environment as a 'singleton' resource coalition i.e. a Cloud with its own internal communication structure, when several 'singleton' resource coalitions agree to share or exchange computing resources (horizontal federation) they behave as if in symbiosis i.e. an autonomous Cloud can request resources from all other Clouds in the same resource coalition. Intuitively, each 'singleton' resource cloud in a resource coalition of size $m$ maintains subscription(s) to each of the remaining $m-1$ resource clouds and exchange messages (communicate) via standard interfaces. Therefore, the worst case communication complexity between resource clouds in a resource coalition of size $m$ is computed as $m(m-1)=m^{2}-m$.

Lemma 1: For resource nodes arranged in ascending (likewise descending) order of resource coalition size for each partition space (level) of the resource graph, the leftmost (rightmost) provider nodes (subspaces) in each partition space $I_{n}$ communicate less via standard interfaces (worst case scenario) in comparison to the rightmost (leftmost) nodes or subspaces.

Proof: If the maximum Resource coalition size of any leftmost (or rightmost) provider node in partition space $I_{2}$ is $m$ then the maximum Resource coalition size of its immediate rightmost (leftmost) sibling node, if any, is at most $m+1$.
Similarly, the minimum resource coalition size of the leftmost (rightmost) node is at most $m$ and the minimum provider coalition size of its immediate rightmost (leftmost) sibling node is at most $(m-1)$.

Hence, we now compare (worst case scenario) the communication complexity of the resource coalitions in the leftmost (rightmost) resource node to its immediate rightmost (leftmost) neighbor in sub space $I_{2}$, hence, we have $2\left(m^{2}-\right.$ $m)<m(m+1)+(m-1)(m-2)$. Which implies, $2 m^{2}-$ $2 m<\left(m^{2}+m\right)+\left(m^{2}-3 m+2\right)$ and $2 m^{2}-2 m<2 m^{2}-$ $2 m+2$. This assertion is true for the base case i.e. sub space $I_{2}$. We also need to prove that this inequality holds for any level (partition space) $I_{n}(n>2)$ in the resource structure graph by reducing the problem in partition space $I_{n}$ to a base case problem (partition space $I_{2}$ ). Generally speaking, we can prove this by looking closely at the pattern that arises in the partition spaces below $I_{2}$ as illustrated in Fig. 3. The pair of leftmost (rightmost) resource nodes and some rightmost (leftmost) neighbor, if any, in Sub space $I_{3}$, have one resource coalition of the same size in common, and in Sub space $I_{4}$, they have two resource coalition sizes in common and so on.

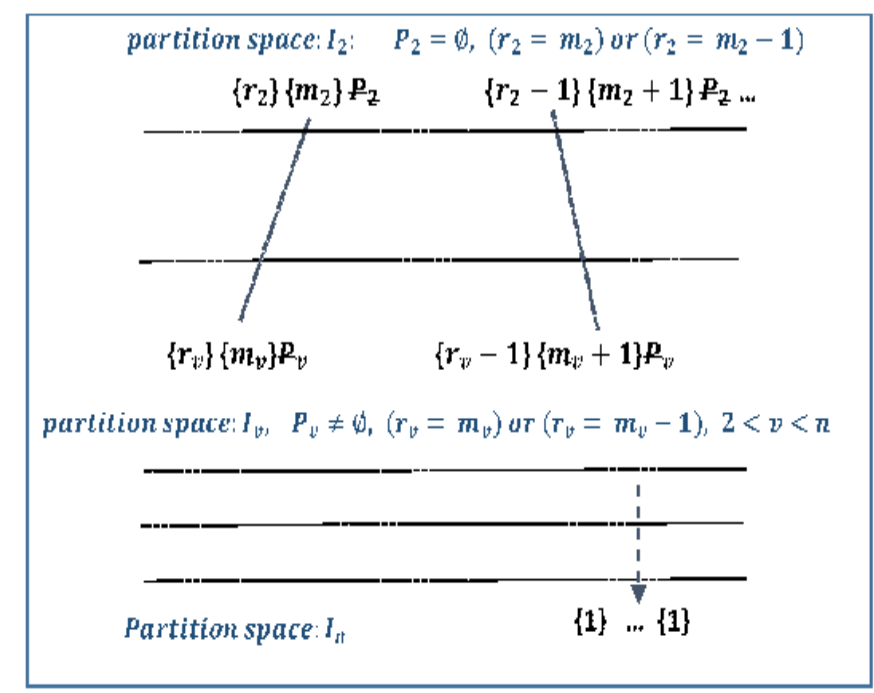

Figure. 3. Schematic representation for reducing resource nodes in level $v, v>2$ to nodes on level 2 .

Therefore, in partition space $I_{n}(n>2)$, comprising more than one provider node, the leftmost (rightmost) resource node and some rightmost (leftmost) neighbor, have $p$ resource coalition sizes in common where $p$ is $n-2$. We can reduce each pair of these nodes by removing the resource coalition sizes they have in common since theoretically these resource coalitions have the same communication complexity in the worst case. In conclusion, resource nodes extant in partition space $I_{n}$ contain $n$ resource coalitions and each pair of leftmost (rightmost) provider nodes and its immediate rightmost (leftmost) neighbor can be reduced (see Fig. 3) to Cloud nodes similar to those in partition space $I_{2}$.

Lemma 2: In a resource graph, provider (resource) nodes in provider partition space $I_{n}(n \geq 1)$ on average have increased 
communication in comparison (worst case analysis) to provider nodes in partition space $I_{n+1}$.

Proof: In ascending (descending) order the rightmost (leftmost) provider nodes in each partition space communicate the most according to lemma 1. Specifically, partition spaces $I_{n+1}$ have a rightmost (leftmost) provider node of the form

$\{1\}_{n}\{1\}\{m-1\}$, where $m+n=k$ and $\{1\}_{n}$ is $n(n \geq 0)$ 'singleton' resource coalitions, i.e. $\{1\}\{1\} \ldots \times n$, then in partition space $I_{n}$ the equivalent rightmost (leftmost) node has the form $\{1\}_{n}\{m\}$. These rightmost (leftmost) provider nodes contain resource coalitions that communicate the most (following the proof of lemma 1) in partition spaces $I_{n+1}$ and $I_{n}$ respectively. Hence, we perform the worst case comparison of the communication complexity as shown in Fig. 4 for provider nodes in partition spaces $I_{n}$ and $I_{n+1}$ following lemma 2, we can say $\left(m^{2}-m\right)>\left(m^{2}-3 m+3\right)$. Since we are strictly dealing with integers, this implies $m>1$, hence, the Inequality proves our assertion is true for rightmost (leftmost) provider nodes in all partition spaces. In addition, it can be shown graphically that the communication complexity of the leftmost (rightmost) provider node in space $I_{n}$ is greater than leftmost (rightmost) node in partition space $I_{n+1}$.

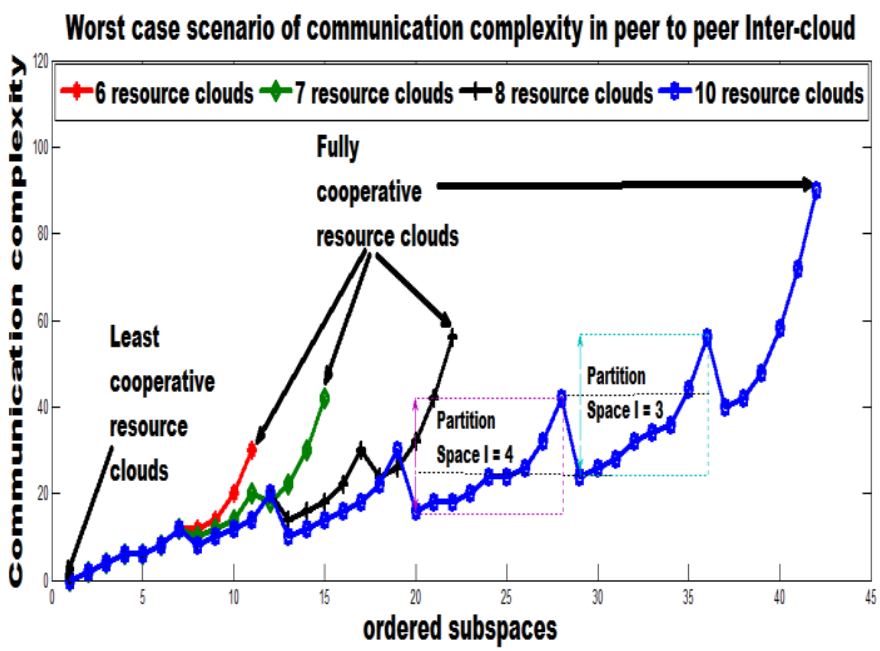

Figure. 4. Worst case analysis of the communication complexity in peer to peer ( $22 \mathrm{p}$ ) InterCloud.

\section{MULTI-CRITERIA OPTIMISATION PROBLEM IN RESOURCE MANAGEMENT FOR PEER TO PEER INTERCLOUD}

To accomplish our goal of finding the most effective and efficient deployment of resources across clouds and prevent a deterioration of InterCloud throughput, we need to employ a test process and make optimal decision in the presence of trade-off between the value (success rates) and communication complexity of resource structures in peer to peer Inter-cloud. The aim is then to find a Pareto optimal solution for the resource structure generation (or CSG) problem comprising the two prominent objectives (value or outcome of resource structures against communication complexity). Intuitively, a decision maker's upmost preference for resource management would be performance (or value) of a resource structure over the complexity of message exchanges (or communication). These preferences must be captured in the solution process for the coalition structure generation problem. Accordingly, we apply a priori multi-criteria optimisation (MCO) method called lexicographic method, which assumes that objectives can be arranged in hierarchy of preference or importance (Jee et al, 2007). Formally, the lexicographic method for the coalition structure generation problem of resource management in peer to peer Inter-cloud consists of two singleobjective optimisation problems:

$R S^{*}=$ Maximize $h_{i}(R S)$

\section{S. t. $R S \in G$}

$h_{j}(R S)=h_{j}^{*}, \quad i=1 \ldots k, j=1 \ldots k-1$ and $k=2$

$h_{1}(R S)=$ Outcome $(R S)$

$h_{2}(R S)=1 / \varphi(R S)$

In applying lexicographic ordering (LO), we denote $h_{1}$ $(R S)$ and $h_{2}(R S)$ as the most preferred and least preferred objective functions respectively. Also, the optimal value(s) $h_{j}{ }^{*}$ $=\arg \max \left(h_{j}(R S)\right)$ is the solution for the single objective function $h_{j}$. Given the partition form game (PFG) for resource management in $\mathrm{p} 2 \mathrm{p}$ InterCloud, consider a resource (or provider) coalition $C$ and a resource coalition structure $R S$ in peer to peer inter-cloud. We denote $\operatorname{outcome}(C, R S)=$ $\left\{\left(N_{\text {succ }}\right)^{R S},\left(N_{A t t^{C}}\right)^{R S}\right\}$, the performance of resource coalition $C$ in $R S$, where $\left(N_{\text {succ }}{ }^{C}\right)^{R S}$ and $\left(N_{A t t}{ }^{C}\right)^{R S}$ is defined as the number of resolved consumer requests (successful) and the number of attempts respectively, achieved by resource coalition $C$ in resource (coalition) structure $R S$. Therefore, the highest priority objective function $h_{1}(R S)$ is computed as Outcome $(R S)=\sum_{C \in R S}$ outcome $(C, R S)=\left\{\sum_{C \in R S}\left(N_{\text {succ }}{ }^{C}\right)^{R S}\right.$, $\left.\sum_{C \in R S}\left(N_{A t t}\right)^{R S}\right\}$ and the least priority $h_{2}(R S)$ is the multiplicative inverse of $\varphi(R S)$, a function which returns the resource structure's communication complexity.

\section{A. IP Algorithm}

The IP algorithm (Rahwan, 2007) is based on an integer partition representation of the search space that groups resource structures into subspaces based on the sizes they contain. What is interesting about this representation is that, it is possible to establish bounds (upper and lower) on the outcome (performance) of the best resource structure in every subspace $P_{I}^{A}$ of $I \in I_{n}$. More precisely, let $M a x_{s}$ and $A v g_{s}$ be the maximum and average outcomes of resource coalitions of size $s(s \leq n)$. For all Integer partitions $I \in I_{n}$, it is possible to compute the upper bound $U B_{I}=\sum_{s \in I} \operatorname{Max}_{s}$ and lower bound $L B_{I}=\sum_{s \in I} A v g_{s}$ respectively. Consequently, these bounds are used to guarantee worst case scenarios on the quality of the best resource structure found so far and also to prune unpromising subspaces. As for the remaining subspaces, they are searched sequentially unless a value is found that is higher than the upper bound of other subspaces, in which case we forfeit searching that subspace. 


\section{B. IP ${ }^{+/-}$Partial search: Minimum search and worst case guarantees}

In a partition form game for resource management with positive and negative externalities, we perform minimum search to establish bound on the optimal resource configuration in peer to peer inter-cloud by observing the maximum (best) outcome of each resource coalition $C$ in some resource coalition structure as conceptualised in Fig. 5. Specifically, if resource degradation is relatively high or common across Clouds $\left(\mathrm{PFG}^{+}\right.$setting), every Integer partition $G_{I}: I \in I_{n}:|I| \leq 2$ must be searched and the number of resource structures searched (or search cost) is $2^{n^{-1}}$ with a worst case ratio bound $\beta=n$. On the other hand, if Clouds' resource failure is low ( $\mathrm{PFG}^{-}$setting), It is necessary to search every partition $G_{I}: I \in\{[n],[n-1,1],[n-2,1,1], \ldots,[1,1, \ldots, 1]\}$. Here, the number of resource coalition structures searched (or search cost) is $2^{n}-n$ and $\beta=\left\lceil\frac{n}{2}\right\rceil$ (see proof in Rahwan et al (2012, 2015))

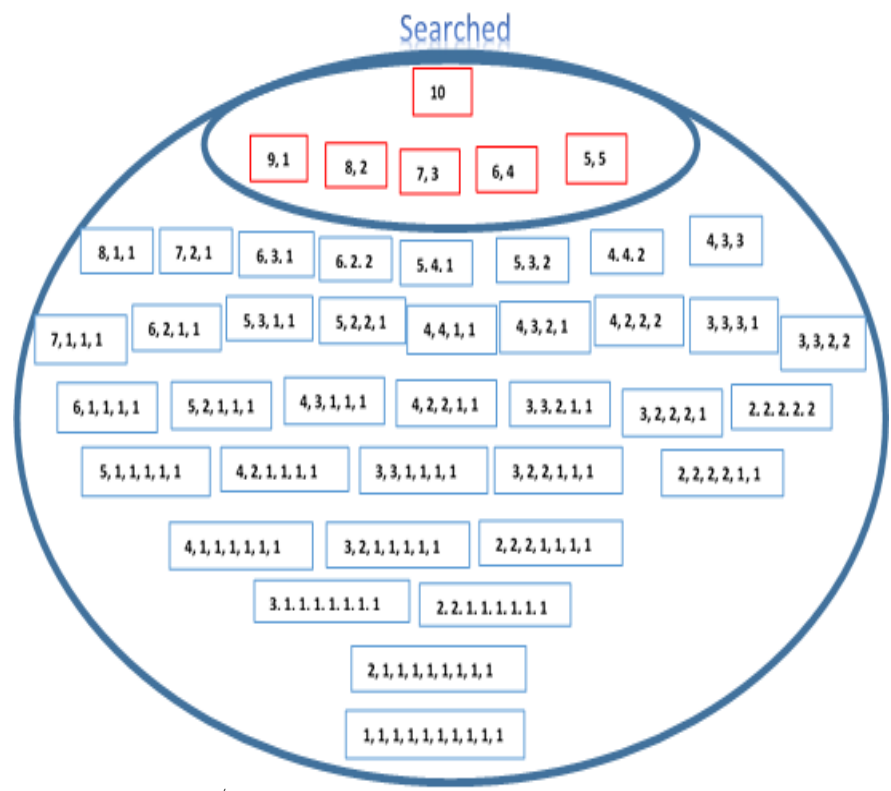

Figure 5. $\mathrm{IP}^{+/-}$partial search for 10 resource clouds when Clouds experience high resource degradation $\left(P F G^{+}\right.$setting)

\section{IP $\quad$ I/- optimal cost anytime algorithm for further} Search

In this subsection we outline our anytime algorithm (uniform cost search) to improve the solution quality with further search. Although Rahwan et al (2012) anytime algorithm for reducing the ratio bound $\beta$ with further search is cost effective, it minimises (using an integer solver) the search cost required to reduce the worst case ratio bound $\beta$ by at least one in each iteration. Their method does not necessarily assume an ordered path of minimal cost. This is because lowering the ratio bound $\beta$ for subspaces of some cost which requires exploring a subspace of higher cost is not exactly cost optimal or cost efficient (see Fig. 6). As a solution, we developed a procedure for further search that determines the Pareto-frontier and is cost-centric, based on the cost of searching subspaces. The search cost or cost of searching an Integer partition is defined as the number of resource structures it contains. For instance: given $n$ resource clouds i.e. (Where $n=10$ ), searching the subspace $P_{I}^{A}$ represented by integer partition $I=[4,3,3]$ incurs a cost $\left|P_{I}^{A}\right|$ of 2100 , equal to the number of constituting resource structures. Our algorithm's prime idea is to search through the remaining subspaces, one after another, ordered by minimum path cost.

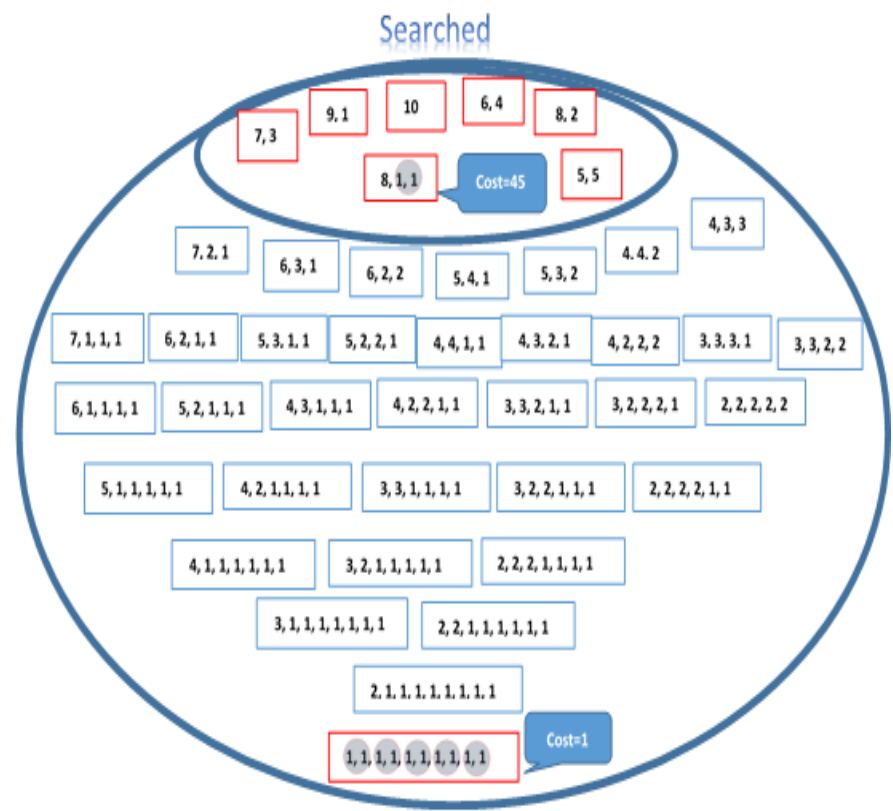

Figure 6. Exploration of a node (with cost $=45$ ) to reduce $\beta$ ( of a partition with cost $=1$ ) from 10 to 5 using $\mathrm{IP}^{+/}$ improving the ratio bound algorithm for 10 resource clouds in $P F G^{+}$setting.

Basically, If we can afford the cost (time), our anytime algorithm uses priority queue as the data structure to find a cost efficient way to improve the solution quality (and lower the bound) by always searching a subset of resource structures with the least cumulative cost from the remaining subspaces. Figure 7 is an illustration (worst case) of the first three minimum cost paths (see callouts) chosen by the proposed algorithm for 10 resource clouds with positive externalities, integers are used to represent coalition sizes. At each path step, our procedure re-computes its search path, that is, the path comprising subspace(s) with the smallest cumulative search cost out of the remaining (unsearched) subspaces. Algorithm 1 presents our three step outline of the proposed anytime algorithm in sequel.

Algorithm 1: $\mathrm{IP}^{+/-}$optimal cost anytime algorithm (Uniform cost search)

1. Perform IP Partial search for PFG

$>$ For negative externalities $\left(\mathrm{PFG}^{-}\right)$: Search

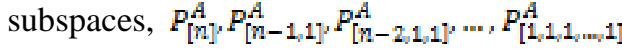

$>$ For Positive Externalities $\left(\mathrm{PFG}^{+}\right)$: Search subspaces $R_{T}^{A}:|I| \leq 2$ 


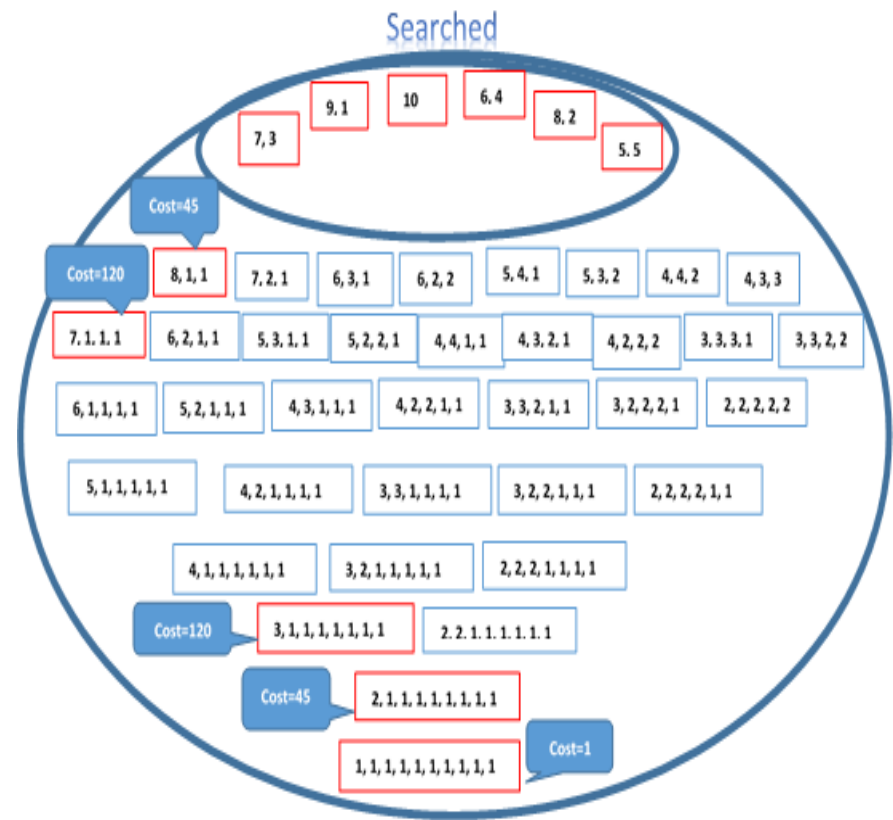

Figure 7. $\mathrm{IP}^{+/-}$optimal cost further search algorithm for 10 resource clouds when Clouds experience high resource degradation $\left(P F G^{+}\right.$setting $)$

2. If there is additional Cost(time) allowance, Continue with Uniform cost search or if the cost permits, minimum cost search the remaining subspaces (resource structures) represented by Integer partitions which involves in worst case not greater than:

$>$ For $\mathrm{PFG}^{+}: P_{I}^{A}: 2<|I| \leq n$

$>$ For $\mathrm{PFG}^{-}$:

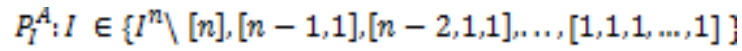

3. Return the best resource structure obtained thus far.

\section{CONCLUSION}

This paper has presented a Self-tuning framework for managing resource in InterCloud, an important and quite novel topic in InterCloud research literature. We have adequately modeled resource management for volunteer (p2p) InterCloud with partition function games in $P F G^{+}$and $P F G^{-}$settings. Using the Integer Partition graph representation, we were able to define a new concept 'resource structure graph', in which nodes at each level of the IP graph must be ordered, in ascending or descending order of the maximum resource coalition size it contained. Interestingly, this representation enabled measurement of worst case communication complexity for any resource coalition of a specific size and any resource structure in peer to peer InterCloud. Accordingly, our research effort has proffered two lemmas (with proof) based on the worst case communication complexity of resource nodes in a resource structure graph. With this background, we were able to identify a multi-objective optimisation problem for resource management and describe its solution formally using a multi-criteria optimisation (MCO) method referred to as lexicographic ordering. Furthermore, we developed an IP-based anytime algorithm, based on uniform cost search, to solve the CSG problem. In future work, this research will pursue empirical evidence in $P F G^{+}$and $P F G^{-}$ settings to measure the efficiency of our proposed anytime algorithm in reducing the ratio bound and improving the solution quality.

\section{ACKNOWLEDGMENT}

This work has been partially supported by Scientific Research Foundation, North China University of Technology and Tertiary Education Trust Fund (TETFUND), Nigeria.

\section{References}

[1] T. Aoyama and H. Sakai (2011). Inter-cloud computing. Business \& Information Systems Engineering 3: 3, 173-177.

[2] M. Armbrust, A. Fox, R. Griffith, A. Joseph, R. Katz (2009). Above the Clouds: A Berkeley View of Cloud Computing. Technical Report, Electrical Engineering and Computer Sciences, University of California at Berkeley.

[3] X. Bai, Y. Chen, \& Z. Shao (2007). Adaptive web services testing. In proceedings of the 31st Annual IEEE International Computer Software and Applications Conference, 2007. COMPSAC 2, 233-236.

[4] A. Banafa (2014). Openmind: Cloud of Clouds (Intercloud). Published: Nov 17 2014. URL https://www.bbvaopenmind.com/en/cloud-clouds-intercloud/\#

[5] D. Bernstein, E. Ludvigson, K. Sankar, S. Diamond and M. Morrow (2009). Blueprint for the InterCloud-protocols and formats for cloud computing interoperability. In Proceedings of the 4th International Conference on Internet and Web Applications and Services. 328-336. IEEE

[6] R. Buyya, R. Ranjan, and R.N. Calheiros (2010). InterCloud: Utility-oriented federation of cloud computing environments for scaling of application services. In Proceedings of the $10^{\text {th }}$ International Conference on Algorithms and Architectures for Parallel Processing (ICA3PP'10), Vol.6081. Busan, South Korea, 13-31.

[7] A. Celesti, F. Tusa, M. Villari and A. Puliafito (2010). How to enhance cloud architectures to enable cross-federation. In Proceedings of the 3rd International Conference on Cloud Computing (Cloud'10). Miami, FL, 337-345.

[8] K. Y. Cai, J.W. Cangussu, R. A. DeCarlo and A. P. Mathur, (2003). An overview of software cybernetics. In proceedings of Eleventh Annual International Workshop on Software Technology and Engineering Practice, 77-86.

[9] D. Chen \& G. Doumeingts (2003). European initiatives to develop interoperability of enterprise applications - basic 
concepts, framework and roadmap. Annual Reviews in Control 27, 2 (2003), 153-162.

[10] A. Chinchuluun. (2008). Pareto Optimality, Game Theory and Equilibria. New York: Springer, Print.

[11] V.D. Dang, \& N.R. Jennings (2004). Generating coalition structures with finite bound from the optimal guarantees. In Proceedings of the Third International Joint Conference on Autonomous Agents and Multi-Agent Systems (AAMAS-04), pp. 564-571.

[12] Global Inter-Cloud Technology Forum. Use Cases and Functional Requirements for InterCloud Computing. Technical Report, Global Inter-Cloud Technology Forum 2010 .

[13] M. Guazzone, C. Anglano, M. Sereno (2014)."A Game-Theoretic Approach to Coalition Formation in Green Cloud Federations," in Cluster, Cloud and Grid Computing (CCGrid), 2014 14th IEEE/ACM International Symposium on ,vol., no., pp.618-625, 26-29 May 2014 doi: 10.1109/CCGrid.2014.37

[14] N. Grozev and R. Buyya (2012). "Inter-Cloud Architectures and Application Brokering: Taxonomy and Survey". Softw. Pract. Exper. 44.3: 369-390. Web.

[15] I. Hafalir (2007). Efficiency in coalition games with externalities. Games and Economic Behavior, 61(2), 242-258. doi:10.1016/j.geb.2007.01.009

[16] B. Horling, \& V. Lesser (2004). A survey of multi-agent organizational paradigms. The knowledge Engineering Review, Cambridge Univ. Press.

[17] K. Jee, D.L. McShan and B.A. Fraass (2007) "Lexicographic Ordering: Intuitive Multicriteria Optimization for IMRT". Physics in Medicine and Biology 52.7: 1845-1861. Web.

[18] J. Lloret, M. Garcia, J. Tomas and J.J. Rodrigues (2014). "Architecture and Protocol for Intercloud Communication". Information Sciences 258: 434-451. Web.

[19] X. Li (2007). Improving multi-agent coalition formation in complex environments (Ph.D). Thesis, Lincoln, NB, USA. AAI3258404

[20] T. Michalak, A. Dowell, P. McBurney and M. Wooldridge (2008). Optimal coalition structure generation in partition function games. In Proceedings of the $18^{\text {th }}$ European conference on Artificial Intelligence (ECAI-08), pages 388392.

[21] T. Rahwan (2007). Algorithms for Coalition Formation in Multi-agent Systems (Ph.D). University of Southampton.

[22] T. Rahwan, T. Michalak, E. Elkind, P. Faliszewski, J. Sroka, M. Wooldridge \& N.R. Jennings (2011). Constrained Coalition Formation. In Proceedings of the Twenty-Fifth AAAI conference on Artificial Intelligence.
[23] T. Rahwan, T. Michalak, M. Wooldridge, \& N. Jennings (2012). Anytime coalition structure generation in multi-agent systems with positive or negative externalities. Artificial Intelligence, 186, 95-122. doi:10.1016/j.artint.2012.03.007

[24] T. Rahwan, S.D. Ramchurn, A. Giovannucci, V.D. Dang, N.R. Jennings (2007). Anytime optimal coalition structure generation, in Proceedings of the Twenty Second Conference on Artificial Intelligence (AAAI), pp1184-1190.

[25] T. Rahwan, T. Michalak, N.R. Jennings, M. Wooldridge, P. McBurney (2009). Coalition structure generation in Multiagent systems with positive and negative externalities, in Proceedings of the Twenty First International joint conference on Artificial Intelligence (IJCAI), Pasadena, USA.

[26] T. Rahwan., Michalak, T. P. McBurney, M. Wooldridge, N.R. Jennings (2015). "Coalition Structure Generation: A Survey". Artificial Intelligence 229: 139-174. Web.

[27] T. Sandholm, K. Larson, M. Andersson, O. Shehory, \& F. Tohmé (1999). Coalition structure generation with worst case guarantees. Artificial Intelligence, 111(1-2), 209-238. doi:10.1016/s0004-3702(99)00036-3

[28] A. N. Toosi, R.N. Calheiros and R. Buyya (2014). Interconnected Cloud Computing Environments: Challenges, Taxonomy, and Survey. ACM Comput. Surv. 47, 1, Article 7 (May 2014), 47 pages. DOI=http://dx.doi.org/10.1145/2593512

[29] D. Villegas , N. Bobroff, I. Rodero, J. Delgado, Y. Liu, A. Devarakonda, L. Fong, S.M. Sadjadi and M. Parashar (2012). Cloud federation in a layered service model. Journal of Computer and System Sciences 78, 5 (2012), 1330-1344. 